\title{
Tumor Necrosis Factor Receptor Mediates Fibroblast Growth Factor-Inducible 14 Signaling
}

\author{
Xuening Wang ${ }^{a}$ Shengxiang Xiao ${ }^{a}$ Yumin Xia ${ }^{a}$ \\ aDepartment of Dermatology, The Second Affiliated Hospital, School of Medicine, Xi'an Jiaotong \\ University, Xi'an, China
}

\section{Key Words}

TWEAK • Fn14 • TRAF • TNFR • Apoptosis • Proliferation • Keratinocyte

\begin{abstract}
Tumor necrosis factor (TNF)-related weak inducer of apoptosis (TWEAK) engages its sole receptor, fibroblast growth factor-inducible 14 (Fn14), which participates in various inflammatory and immunologic processes. TWEAK/Fn14 interaction induces different cell fates depending on the local microenvironment, which correlates with certain expression profiles of TNF receptors (TNFR). The predominant expression of TNFR1 or TNFR2 facilitates cell death or proliferation, respectively, on TWEAK/Fn14 activation. TNFR-associated factors (TRAF) interact with Fn14, cellular inhibitor of apoptosis protein (CIAP)-1, and TNFR, consequently transducing signals from TWEAK to downstream cytokines and cell cycle mediators. An Fn14-TRAF2-TNFR axis has been suggested in the function of TWEAK/Fn14 signaling, which may serve as a target in the development of novel therapeutic strategies for many diseases that have Fn14overexpressing cells in affected tissues. The aims of this review are: 1 ) to present the main results on TWEAK/Fn14 regulation of cell fates, 2) to analyze the mechanism of the Fn14TRAF2-TNFR axis, and 3) to summarize the potential strategies in the pharmacologic targeting of this axis.

\section{Introduction}

Tumor necrosis factor (TNF)-related weak inducer of apoptosis (TWEAK) belongs to the TNF superfamily and acts through binding to its sole receptor, fibroblast growth factor-inducible 14 (Fn14). TWEAK can be originally expressed as a type II transmembrane protein but can be cleaved to form a soluble cytokine [1, 2]. TWEAK is mainly secreted by macrophages/monocytes in inflammatory tissues [3], and is widely expressed in different tissues or organs, including the pancreas, intestines, heart, brain, lungs, ovary, vasculature, 


\section{Cellular Physiology Cell Physiol Biochem 2017;43:579-588 \begin{tabular}{ll|l} 
DOI: 10.1159/000480530 & $\begin{array}{l}\text { O 2017 The Author(s). Published by S. Karger AG, Basel } \\
\text { www.karger.com/cpb }\end{array}$
\end{tabular} \\ Wang et al.: Fn14-TRAF2-TNFR Axis}

and skeletal muscles, and has low expression in the liver and kidney [4]. Fn14, a type I transmembrane protein [5], has been reported to be expressed in the nervous system, such as in microglia and astrocytes, and in immune cells, including natural killer cells, macrophages, dendritic cells, and Th17 cells [6]. Besides, Fn14 can be expressed in normal keratinocytes, immortal HaCaT cells, or keratinocytes under different inflammatory conditions [7-13]. In healthy tissues, the expression levels of TWEAK and Fn14 are relatively low [6]; however, their expression increases when the tissues are damaged or under inflammation [14].

In recent years, increasing evidence has indicated that the TWEAK/Fn14 signaling pathway plays a pivotal role in the pathogenesis of lupus erythematosus, which presents cutaneous, renal, and neuropsychiatric diseases [15-17]. Moreover, the TWEAK/Fn14 signals participate in the development of various dermatoses, such as psoriasis [7], atopic dermatitis [8], cutaneous vasculitis [18], human papillomavirus (HPV) infection [9], and bullous pemphigoid [10]. TWEAK/Fn14 activation also contributes to the biological processes of tumors [19]. TWEAK is a significant proinflammatory cytokine in vitro and in vivo [20]. TWEAK/Fn14 interaction has been shown to mediate diverse bioactivities involving the activation of cell proliferation, migration, or apoptosis; the induction of vasculitis and angiogenesis; the production of inflammatory cytokines; and the promotion of fibrogenic responses $[15,20]$. In in vitro experiments, TWEAK has been found to induce the production of multiple cytokines, including regulated on activation, normal T-cell expressed and secreted (RANTES), monocyte chemoattractant protein-1 (MCP-1), interferon- $\gamma$-induced protein 10 (IP-10), macrophage inflammatory protein-1 alpha (MIP-1 $\alpha$ ), intercellular adhesion molecule-1 (ICAM-1), and vascular cell adhesion molecule-1 (VCAM-1) [14, 21]. In addition, TWEAK has the capacity to induce the production of matrix metalloproteinases and inflammatory mediators (RANTES and IP-10) in human dermal or synovial fibroblasts [14]. TWEAK also promotes the synthesis of RANTES, MCP-1, IP-10, MIP-1 $\alpha$, ICAM-1, and VCAM-1 in cutaneous keratinocytes $[12,21]$. These cytokines accumulate in local injured tissues and trigger immune responses and even exacerbate inflammatory responses.

The inflammatory responses in various diseases are complicated and are accompanied by different fates of resident cells. Different types of cells may proliferate or undergo apoptosis (or necrotize) under certain inflammatory conditions. For instance, TWEAK/Fn14 activation promotes the proliferation of mesangial cells, as well as the death of tubular cells, glomerular endothelial cells, and podocytes in kidneys under lupus inflammation [22, 23]. Moreover, the same types of cells show diverse cell cycles under various microenvironments. Further, TWEAK/Fn14 signals are involved in cutaneous inflammation and diversely regulate the cell fates of keratinocytes [7-11]. In fact, the regulation of cell fates is central to the function of TWEAK/Fn14 signaling. Why and how does TWEAK/Fn14 activation lead to different cell fates? This review summarizes the recent findings and attempts to find some clues or answers.

\section{TWEAK/Fn14 activation modulates different cell fates}

TWEAK/Fn14 activation can induce the proliferation of cells. However, it can also induce cell death (apoptosis or necrosis). The cell fates of proliferation and death are clearly diametrically opposed. These phenomena have been evidenced in many studies and currently attract considerable attention.

Based on convincing experiments, TWEAK is originally defined as a weak inducer of apoptosis [1]. This is somehow inconsistent with the fact that Fn14 does not have a death domain [14]. Given that there is no death domain in Fn14, it is speculated that TWEAK may use TNF receptor-associated factor (TRAF) type 3-mediated induction of apoptotic complex formation, which leads to LT $\beta$ receptor-triggered cell death [14]. The precise mechanisms by which the exact signals are transduced in TWEAK-induced apoptosis seem to involve multiple processes, including TNF- $\alpha$ or caspase-dependent (or -independent) death, with the characteristics of both apoptosis and necrosis [14, 24-26]. TWEAK and TNF- $\alpha$ cooperate 
in the induction of apoptosis in human keratinocytes from healthy subjects, patients with atopic dermatitis, and patients with psoriasis, as well as in keratinocyte cell lines [8]. TWEAKinduced cell death also involves cathepsin B-dependent necrosis [13]. TWEAK stimulation leads to massive activation of apoptotic cell death, characterized by activation of the initiator proteases caspase- 3 and caspase-7, processing of the initiator proteases caspase- 8 and caspase-9, and induction of mitochondrial outer membrane permeabilization [27]. Similarly, a study of Kym1, SKOV3, and OVCAR4 cell lines has shown that TWEAK triggers cell death indirectly through autocrine TNF- $\alpha$ signaling [26].

Although there is strong evidence in support of TWEAK as an apoptosis inducer, TWEAK also serves as a growth factor for many cell types, such as synoviocytes, astrocytes, vascular cells, and mesangial cells $[14,28]$. TWEAK not only induces cell growth, which contributes to tissue repair after acute injuries, but also plays a critical role in pathologic hyperplasia involving arthritis (pannus formation), colitis (crypt epithelization), neurodegenerative diseases (glial hyperplasia), and cancers [14]. TWEAK/Fn14 interaction activates classic nuclear factor kappa-light-chain-enhancer of activated B cells (NF- $\kappa B$ ) signals, which are suggested to enhance RANTES expression and macrophage infiltration [7]. NF- $\kappa B$ signals are pivotal in the differentiation, proliferation, and survival of cells, as well as the development of tumors, with the latter also associated with inflammation [29]. The NF- $\mathrm{B}$-activated proliferation marker Ki-67 and antiapoptotic proteins, such as c-Myc, survivin, cIAP-2, and cellular FLICE-inhibitory protein (cFLIP), are upregulated in keratinocytes on TWEAK stimulation [7]. cFLIP is highly expressed in psoriatic tissues [30] and is even promoted by TWEAK in keratinocytes that originate from psoriatic tissues [7]. TWEAK is abundantly expressed in HPV-infected skin warts, and induces the proliferation of keratinocytes transfected with HPV16 E6/E7 genes [9]. Therefore, TWEAK/Fn14 activation enhances the proliferation of cells under certain inflammatory conditions.

\section{Inflammatory microenvironments influence the effect of TWEAK on cell fate}

Both TWEAK and Fn14 are highly expressed in various cutaneous inflammatory disorders, including lupus erythematosus [11, 31], psoriasis [7], atopic dermatitis [8], skin warts [9], and bullous pemphigoid [10]. The downstream cytokines, such as RANTES, MACP-1, IP-10, MIP-1 $\alpha$, ICAM-1, and VCAM-1, are produced locally, reflecting the activation of the TWEAK/Fn14 pathway [7-11, 21, 32]. These disorders not only present different characteristics of skin inflammation but also have cutaneous keratinocytes with diverse fates. In fact, TWEAK affects cell fate depending on the local microenvironments. Here we summarize the effect of TWEAK on identical cells (keratinocytes) under different cutaneous inflammatory conditions.

TWEAK/Fn14 interaction induces death (apoptosis and necrosis) of keratinocytes in atopic dermatitis and cutaneous lupus erythematosus [8, 11, 31]. Atopic dermatitis is characterized by the production of T helper 1 (Th1) cytokines, including interferon- $\gamma$ in lesional skin [32]. Other cytokines, such as IP-10, TNF- $\alpha$, and interleukin (IL)-18, are also synthesized by keratinocytes in affected tissues [32]. Interferon- $\gamma$ induces the apoptosis of keratinocytes in patients with atopic dermatitis [33]. Moreover, TWEAK alone induces the apoptosis of keratinocytes originating from affected skin, which can be exaggerated by TNF- $\alpha$ [8]. The inflammation in cutaneous lupus erythematosus is also predominantly mediated by Th1 cytokines, including interferon- $\gamma$ and IP-10 [34]. Besides, IL-1, IL-6, IL-17, and TNF- $\alpha$ are also produced in cutaneous lupus erythematosus [34]. Ultraviolet B irradiation induces the keratinocyte release of IL-1 and TNF- $\alpha$, two primary cytokines in the inflammatory cascade [34]. Ultraviolet B also acts synergistically with IL-1 $\alpha$ to promote the production of TNF- $\alpha$, which can increase its own production in an autocrine manner [34]. This cytokine cascade recruits immune cells, exacerbates inflammation, induces the apoptosis of keratinocytes, and ultimately destroys skin tissues, resulting in the photo-induced lesions of cutaneous lupus erythematosus [34]. TWEAK/Fn14 activation is prominent in skin lesions of patients 
with lupus erythematosus and in MRL/lpr lupus-prone models [11, 31]. Fn14 deficiency in MRL/lpr mice attenuates ultraviolet B irradiation-induced skin lesions and apoptosis of cutaneous keratinocytes $[11,31]$. Apoptosis of keratinocytes is also prominent in skin lesions of bullous pemphigoid [35]. Moreover, TWEAK/Fn14 activation has been found to be remarkable in skin lesions of patients with bullous pemphigoid and to contribute to loss of BP180 and cellular adherence, which are central to the pathogenesis of bullous pemphigoid [10].

However, TWEAK/Fn14 activation prefers to induce the proliferation of keratinocytes under inflammation of psoriasis and HPV infection [7, 9]. Psoriasis is most likely a Th1/Th17-induced inflammatory disease [36] and is characterized by overexpressing cytokines, such as IL-2, IL-17, IL-23, interferon- $\gamma$, and TNF- $\alpha[7,36]$. Both TWEAK and Fn14 are highly expressed in skin lesions of patients with psoriasis vulgaris, and the Fn14 expression in cultured keratinocytes is enhanced by the M5 cocktail of cytokines (IL1a, IL-17A, IL-22, oncostatin M, and TNF- $\alpha$ ), which induces inflammation that presents several features of psoriasis [7]. TWEAK, in combination with M5 cytokines, promotes the proliferation of keratinocytes that originate from either healthy donors or patients with psoriasis [7]. Additionally, TWEAK enhances the synthesis of survivin, cIAP2, and cFLIP in keratinocytes under psoriatic inflammation [7]. HPV infection of keratinocytes in anogenital regions is known to be associated with a strong etiology of carcinogenesis [37]. Recently, we found that keratinocytes express more Fn14 in HPV16-positive skin warts or in keratinocytes transfected with HPV16 E6/E7 genes [9]. The E6/E7 oncogenes of HPV endow keratinocytes with the property of immortalization by causing genomic instability, favoring additional mutations, and reactivating cell cycle progression in DNA synthesis [9, 38]. Moreover, TWEAK/Fn14 activation is prominent in warts due to the accumulation of TWEAK and downstream cytokines, including RANTES, MCP-1, and IP-10, in affected skin tissues [9]. HPV16 infection itself promotes the proliferation of keratinocytes, as well as switches keratinocytes from apoptotic to proliferative fate on TWEAK/Fn14 activation [9]. HPV infection triggers inflammatory responses that are characterized by an upregulated expression of Th2 cytokines [39]. The expressions of IL-1 $\beta$, IL-4, IL-6, IL-10, interferon- $\gamma$, and TNF- $\alpha$ increase in skin of transgenic mice expressing the HPV16 E7 oncoprotein within basal keratinocytes [39]. However, whether these cytokines participate in the TWEAKinduced proliferation of keratinocytes remains unclear.

In general, the TWEAK/Fn14 pathway is activated in various cutaneous diseases that present different profiles of cytokines in affected skin tissues. The inflammatory microenvironments may generate diverse cell phenotypes, which may further influence the effect of TWEAK on these cells.

\section{Switch in cell phenotype reverses the direction of TWEAK regulation}

TNF- $\alpha$ is currently understood to interact with two receptors: TNFR1 and TNFR2 [8]. TNF can be divided into soluble and membranous types [40]. Soluble TNF predominantly activates TNFR1, whereas membranous TNF can combine with and effectively activate both TNFR1 and TNFR2 [40]. Actually, compared with soluble TNF, membranous TNF exhibits higher affinity in engaging TNFR2 [41]. The distribution of TNFR1 and TNFR2 also differs in tissues. TNFR1 is ubiquitously expressed, whereas TNFR2 is more restrictedly expressed, mainly in immune cells [42]. However, TNFR2 is also expressed in some cancer cells and cutaneous keratinocytes $[8,43,44]$. TNFR1 may induce cell death, whereas TNFR2 does not induce cell death directly $[40,45]$. TNFR2 may enhance cytotoxicity mediated by TNFR1 when TNFR1 and TNFR2 are activated simultaneously $[40,45]$. Further, TNFR2 promotes cell proliferation and survival by leading the transcriptional activation of the proliferationrelated genes, which is mediated by TRAF2 [40].

TNFR1 has a death domain in its intracellular region [40]. The TNFR1 signaling pathway is activated by the signals of apoptosis or necroptosis, which are contingent on 
the environments and cellular states [46]. TNFR1 signaling either induces the synthesis of multiple inflammatory mediators and growth factors through NF- $\mathrm{BB}$ activation or can result in the induction of cell death through its death domain through the TNF receptor-associated protein with death domain or the Fas-associated protein with death domain, which mediates cell death signaling $[42,47]$.

In contrast to TNFR1, TNFR2 does not have a death domain [40]. TNFR2 activates $\mathrm{NF}-\kappa \mathrm{B}$ and $\mathrm{c}$-Jun N-terminal kinase and then causes transcriptional activation of the genes that are associated with cell proliferation and survival [45]. NF- $\kappa B$ signaling leads to the transcription of antiapoptotic genes, such as cIAP1, cIAP2, cFLIP, TRAF1, and TRAF2 [40]. TNFR2 also activates the noncanonical NF- $\kappa B$ pathway, which participates in the differentiation and survival of cells [40]. Moreover, Fn14 and TNFR2, which belong to a subset of TNFR superfamily members, can also characteristically activate the noncanonical NF- $\kappa B$ pathway [48]. The RING finger protein TRAF2 plays a key role in TNFR1- and TNFR2induced signaling. TRAF2 can be recruited to TNFR1 or TNFR2 and even mediates the cross talk between TNFR1 and TNFR2 [46]. TNFR2 activation also induces the degradation of TRAF2 [45].

Recent studies have shown that TWEAK regulates cell fate differently depending on the TNFR subtypes in keratinocytes [7-9, 11, 13]. TNFR1 is predominantly expressed in normal keratinocytes or in keratinocytes under inflammation of lupus erythematosus or atopic dermatitis $[8,11,13]$. However, psoriatic inflammation or HPV infection favors the expression of TNFR2 in keratinocytes [7, 9]. Accordingly, TWEAK/Fn14 interaction induces keratinocyte death in skin lesions of lupus erythematosus or atopic dermatitis $[8,11]$ but promotes keratinocyte proliferation in psoriatic skin or on HPV infection [7, 9]. Additionally, TWEAK induced TNF- $\alpha$ secretion and TNFR1-dependent convergence of a death-signaling complex involved in receptor-interacting protein 1, Fas-associated protein with death domain, and caspase-8, subsequently inducing caspase-8 activation and cell apoptosis [27]. Similarly, TWEAK/Fn14 interaction may trigger the degradation of cIAPs and then enhances apoptotic processes through TNFR1 [27]. The expression profile of TNFR1/2 clearly correlates with certain inflammatory conditions and influences the effect of TWEAK on keratinocytes. The inflammatory microenvironments may generate diverse cell phenotypes, which may further affect the TWEAK regulation of cell fate. However, the precise mechanism by which TWEAK triggers proliferation signals in cells through TNFR2 remains unclear and needs further investigation.

\section{Structural basis for the interaction between Fn14, TRAF2, cIAP1, and TNFR}

An intracellular signal transduction that may explain the TWEAK regulation of cell fate may be speculated. First, TWEAK binds to its sole receptor Fn14, recruiting the TRAF2cIAP1 complex with Fn14. Then, the TRAF2-cIAP1 complex activates the noncanonical NF$\kappa B$ pathway. Finally, the TRAF-cIAP1 complex can bind to TNFR1 or TNFR2, triggering death or proliferation signals in cells. That is, an Fn14-TRAF2-TNFR axis regulates intracellular signal transduction, which further affects the fate of cells.

TWEAK is a glycoprotein containing a total of 249 amino acids in three parts: the C-terminal extracellular region has 206 amino acids, the transmembrane domain has 25, and the N-terminal intracellular region has 18 [24]. The smallest receptor among the TNFR superfamily members identified thus far is Fn14, which is only 102 amino acids in length; this includes the extracellular domain (53 amino acids), which contains the TWEAK binding site, and the cytoplasmic tail region (28 amino acids), which is essential for signal transduction [19]. TWEAK and Fn14 are the only ligand and receptor for each other among the TNF/TNFR superfamily members [19]. The cytoplasmic signaling pathways start at the cytoplasmic tail of Fn14, which contains a single TRAF binding site; TRAF1, TRAF2, TRAF3, and TRAF5 are all capable of combining with this motif [49]. The Fn14-triggered effect requires a complete TRAF binding motif in the Fn14 cytoplasmic tail $[14,49,50]$. In addition, TRAF2 can also 
bind to TNFR1 or TNFR2, which recruits TRAF2 when stimulated by TNF- $\alpha$, thereby inducing downstream signals [42]. However, although the two receptors both combined with TRAF2, TNFR1- and TNFR2-induced signals are usually divergent in cell fate [46].

TWEAK combines with Fn14 to recruit both TRAF2 and cIAP1. This indicates that endogenous cIAP1 binds to TRAF2, as well as combines indirectly with Fn14 by associating with TRAF2 [26]. Fn14 signaling induces the lysosomal degradation of cIAP1-TRAF2 in a cIAP1-dependent way. This is not similar to the IAP antagonists that cause rapid proteasomal degradation of cIAP1 [26]. There are three baculovirus IAP repeat regions in cIAP1, in which the IAP repeat region 1 domain is bound to TRAF [29]. There are TRAF-N and TRAF-C domains in TRAF2. The TRAF- $\mathrm{N}$ domain with coiled-coils combines with cIAP1/2, whereas the TRAF-C domain has the binding site to TNF receptors, such as TNFR2 [29]. TWEAK reduces the cIAP1-TRAF2 complex, leading to immortalized and minimally passaged tumor cells that are sensitive to TNF- $\alpha$, whereas the primary cells remain resistant [26]. In contrast, overexpression of the cIAP1-TRAF2 complex limits Fn14 signaling and preserves tumor cells from TNF- $\alpha$ sensitization induced by TWEAK [29]. Therefore, TWEAK/Fn14 interaction induces lysosomal degradation of cIAP1-TRAF2, which may accurately affect the balance of proliferation/death signals originating from TNF receptors in cells [26, 51]. TRAF1 and TRAF2 have been initially identified to be associated with cIAP1/2, and then bind to the cytoplasmic domain of TNFR2 $[26,29]$.

Some issues on the Fn14-TRAF2-TNFR axis remain unclear and require experimental studies for verification. For example, how are TRAF2 and Fn14 combined, and what is the exact effect of TRAF2 on the downstream pathways? Figure 1 shows a diagram of the Fn14TRAF2-TNFR axis.

\section{Application of the Fn14-TRAF2-TNFR axis in therapeutic strategies}

The Fn14-TRAF2-TNFR axis can activate the noncanonical NF-кB pathway, which regulates cell proliferation, angiogenesis, and inflammatory cell infiltration. Therefore, the Fn14-TRAF2-TNFR axis is believed to participate in the pathogenesis of various inflammatory diseases and tumors, and blocking any part of this axis may represent a new strategy for treating such disorders.

There are multiple approaches to preventing the activation of the Fn14-TRAF2-TNFR axis in tissues. Both an anti-TWEAK neutralizing antibody and a soluble Fn14-Fc decoy protein can be used to prevent TWEAK from combining with Fn14 in cells [50, 52]. In

Fig. 1. Diagram of the Fn14TRAF2-TNFR axis. TWEAK binds to Fn14, recruiting the TRAF2-cIAP1 complex with Fn14. TRAF2 can also be combined with TNFR. The activation of the death domain of TNFR1 triggers its binding to the TNF receptor-associated protein with death domain (TRADD) and activates caspase 8, thereby promoting apoptosis. In contrast, when TRAF2 binds to TNFR2, the signals of cell proliferation are activated through the NF- $\mathrm{KB}$ and c-Jun $\mathrm{N}$-terminal kinase (JNK) pathways.

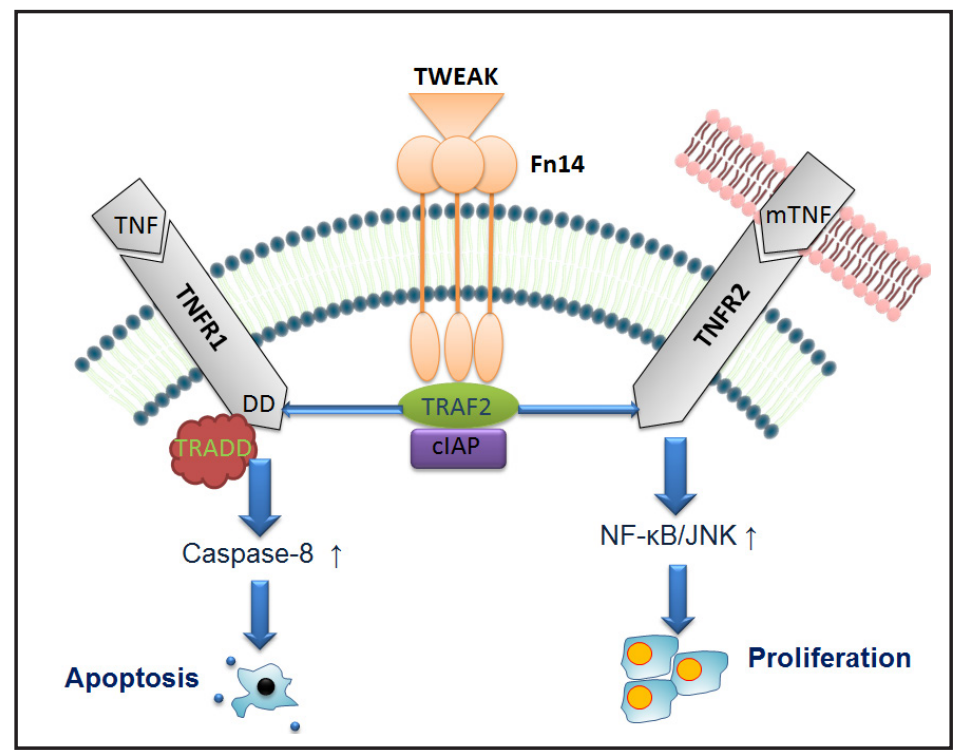


fact, the therapeutic efficacy of using these two agents in vivo has been remarkable [50]. Glomerular immunoglobulin deposition, macrophage infiltration, and tubulointerstitial fibrosis were significantly reduced in lupus nephritis-prone mice treated with an antiTWEAK antibody [22]. The mechanism of such action is highly possible due to a decrease in the downstream target of TWEAK/Fn14 signaling, including decreased expressions of RANTES, MCP-1, IP-10, VCAM-1, and other fibrogenic molecules in kidney [22]. Other strategies use antibodies to the anti-Fn14 extracellular region or small-molecule antagonists to prevent Fn14 trimerization [50]. Blockade of Fn14 trimerization, TRAF-Fn14 association, or downstream pathway activation may also be feasible [50]. In MRL/lpr lupus-prone mice, Fn14 deficiency can significantly attenuate cutaneous disease and the skin infiltration of $\mathrm{T}$ cells and macrophages [11]. Therapies targeting TWEAK/Fn14 interaction can potentially prevent pathologic remodeling and fibrosis but allow endogenous tissue repair. Even with the biological regulation of progenitor cells, inhibition of the TWEAK/Fn14 pathway may promote the regeneration potential of tissues [53].

TRAF2-dependent signaling plays a key role in inflammation, innate immunity, and cell survival [29]. Therefore, TRAF2 can be used as a pharmacologic target to treat autoimmune diseases and cancers. One possible approach to such therapeutic intervention is the destruction of the cIAP1/2-TRAF2 association [29]. Newly identified binding sites that combine TRAF2 with cIAP1/2 enable this strategy to work [54]. If the degradation of TRAF2 is blocked, the TNFR2 activation of the noncanonical NF- $\kappa B$ pathway may be eliminated, possibly leading to T-cell dysregulation. Inducing, instead of blocking, TRAF2 degradation is favorable for cell apoptosis and in some cases can be beneficial as an anticancer strategy [46]. Multimerization of anti-Fn14 antibodies mediated by an Fc receptor may promote the efficiency of their agonistic activity, leading to a more efficient induction of cell death [55]. Some studies have found evidence of the antitumor function of TWEAK/Fn14 signaling, and other works have used Fn14 agonistic agents to verify this potential [55].

A fusion protein of the Fn14-TNF-related apoptosis-inducing ligand (TRAIL) has been found to target two remarkable intercellular signaling axes: TWEAK-to-Fn14 and TRAILto-TRAIL receptor (TRAILR) [56]. This fusion protein was originally designed to inhibit autoimmunity [57]. As a TWEAK blocker, it reduces the permeability of the blood-brain barrier and the transport of inflammatory cells to the central nervous system, showing excellent efficacy in an autoimmune encephalomyelitis model [56]. Fn14-TRAIL has also been shown to inhibit the growth of hepatocellular carcinoma cells in vitro and in vivo [57]. This fusion protein has an effective antitumor activity given that it targets two signal axes simultaneously. Zhou et al. generated two chemical conjugates, ITEM4-rGel and hSGZ [58]: ITEM4-rGel consists of rGel toxin and an anti-Fn14 antibody, whereas hSGZ is a humanized dimeric single-chain antibody conjugated with rGel. Both ITEM4-rGel and hSGZ are extremely cytotoxic to different groups of melanoma cell lines. These two immunotoxins induce melanoma cell necrosis and upregulate the expression of Fn14 in cells [58]. Therefore, Fn14 can be used as a new therapeutic target for melanoma treatment.

The use of the Fn14-TRAF2-TNFR axis presents a promising therapeutic approach. Future investigations should focus on the potential role of the TWEAK/Fn14 pathway in different diseases, as well as consider the disease activities, patient subsets, and interactions with other crucial immunologic pathways [53].

\section{Conclusion}

The Fn14-TRAF2-TNFR axis transduces intracellular signals in the TWEAK/Fn14 pathway. TWEAK/Fn14 activation shows diverse cell cycles depending on the predominance of TNFR1 or TNFR2 expression, which is mainly affected by the local inflammatory microenvironments. The activation of TNFR1 and TNFR2 signals induces cell proliferation or apoptosis, respectively. By targeting the Fn14-TRAF2-TNFR axis, novel therapeutic approaches may be developed for the treatment of chronic inflammatory diseases and cancers. 


\section{Cellular Physiology Cell Physiol Biochem 2017;43:579-588

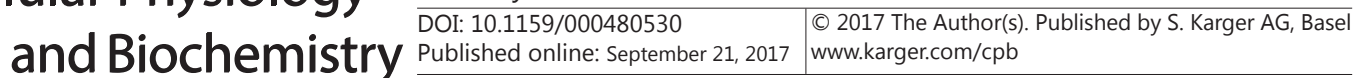 \\ Wang et al.: Fn14-TRAF2-TNFR Axis}

\section{Acknowledgments}

This study was supported by the National Natural Science Foundation of China (Projects No.81472876 and No.81630081) and the Fundamental Research Funds for the Central Universities (No.2015qngz01).

\section{Disclosure Statement}

The authors declare no conflict of interests.

\section{References}

$\checkmark 1$ Chicheportiche Y, Bourdon PR, Xu H, Hsu YM, Scott H, Hession C, Garcia I, Browning JL: TWEAK, a new secreted ligand in the tumor necrosis factor family that weakly induces apoptosis. J Biol Chem 1997;272:32401-32410.

-2 Brown SA, Ghosh A, Winkles JA: Full-length, membrane-anchored TWEAK can function as a juxtacrine signaling molecule and activate the NF-kappaB pathway. J Biol Chem 2010;285:17432-17441.

-3 Bird TG, Lu WY, Boulter L, Gordon-Keylock S, Ridgway RA, Williams MJ Taube J, Thomas JA, Wojtacha D, Gambardella A, Sansom OJ, Iredale JP, Forbes SJ: Bone marrow injection stimulates hepatic ductular reactions in the absence of injury via macrophage-mediated TWEAK signaling. Proc Natl Acad Sci USA 2013:110:6542-6547.

4 Leng RX, Pan HF, Qin WZ, Wang C, Chen LL, Tao JH, Ye DQ: TWEAK as a target for therapy in systemic lupus erythematosus. Mol Biol Rep 2011;38:587-592.

5 Bossen C, Ingold K, Tardivel A, Bodmer JL, Gaide O, Hertig S, Ambrose C, Tschopp J, Schneider P: Interactions of tumor necrosis factor (TNF) and TNF receptor family members in the mouse and human. J Biol Chem 2006;281:13964-13971.

6 Sanz AB, Izquierdo MC, Sanchez-Nino MD, Ucero AC, Egido J, Ruiz-Ortega M, Ramos AM, Putterman C, Ortiz A: TWEAK and the progression of renal disease: clinical translation. Nephrol Dial Transplant 2014;29 Suppl 1:i54-i62.

7 Cheng H, Xu M, Liu X, Zou X, Zhan N, Xia Y: TWEAK/Fn14 activation induces keratinocyte proliferation under psoriatic inflammation. Exp Dermatol 2016;25:32-37.

8 Zimmermann M, Koreck A, Meyer N, Basinski T, Meiler F, Simone B, Woehrl S, Moritz K, Eiwegger T, Schmid-Grendelmeier P, Kemeny L, Akdis CA: TNF-like weak inducer of apoptosis (TWEAK) and TNF-alpha cooperate in the induction of keratinocyte apoptosis. J Allergy Clin Immunol 2011;127:200-207, 207 e201210.

9 Cheng H, Zhan N, Ding D, Liu X, Zou X, Li K, Xia Y: HPV type 16 infection switches keratinocytes from apoptotic to proliferative fate under TWEAK/Fn14 interaction. J Invest Dermatol 2015;135:2427-2436.

10 Liu Y, Peng L, Li L, Liu C, Hu X, Xiao S, Xia Y: TWEAK/Fn14 activation contributes to the pathogenesis of bullous pemphigoid. J Invest Dermatol 2017;137:1512-1522.

11 Doerner JL, Wen J, Xia Y, Paz KB, Schairer D, Wu L, Chalmers SA, Izmirly P, Michaelson JS, Burkly LC, Friedman AJ, Putterman C: TWEAK/Fn14 signaling involvement in the pathogenesis of cutaneous disease in the MRL/lpr model of spontaneous lupus. J Invest Dermatol 2015;135:1986-1995.

12 Jin L, Nakao A, Nakayama M, Yamaguchi N, Kojima Y, Nakano N, Tsuboi R, Okumura K, Yagita H, Ogawa H: Induction of RANTES by TWEAK/Fn14 interaction in human keratinocytes. J Invest Dermatol 2004;122:1175-1179.

-13 Sabour Alaoui S, Dessirier V, de Araujo E, Alexaki V, Pelekanou V, Lkhider M, Stathopoulos EN, Castanas E, Bagot M, Bensussan A, Tsapis A: TWEAK affects keratinocyte G2/M growth arrest and induces apoptosis through the translocation of the AIF protein to the nucleus. PLoS One 2012;7:e33609.

-14 Burkly LC, Michaelson JS, Hahm K, Jakubowski A, Zheng TS: TWEAKing tissue remodeling by a multifunctional cytokine: role of TWEAK/Fn14 pathway in health and disease. Cytokine 2007;40:1-16.

15 Chen J, Wei L, Xia Y: Roles of tumour necrosis factor-related weak inducer of apoptosis/fibroblast growth factor-inducible 14 pathway in lupus nephritis. Nephrology (Carlton) 2017;22:101-106.

16 Wen J, Doerner J, Weidenheim K, Xia Y, Stock A, Michaelson JS, Baruch K, Deczkowska A, Gulinello M, Schwartz M, Burkly LC, Putterman C: TNF-like weak inducer of apoptosis promotes blood brain barrier 


\section{Cellular Physiology Cell Physiol Biochem 2017;43:579-588 \begin{tabular}{l|l|l|} 
DOI: 10.1159/000480530 & $\begin{array}{l}\text { O 2017 The Author(s). Published by S. Karger AG, Basel } \\
\text { www.karger.com/cpb }\end{array}$
\end{tabular} \\ Wang et al.: Fn14-TRAF2-TNFR Axis}

disruption and increases neuronal cell death in MRL/lpr mice. J Autoimmun 2015;60:40-50.

17 Wen J, Xia Y, Stock A, Michaelson JS, Burkly LC, Gulinello M, Putterman C: Neuropsychiatric disease in murine lupus is dependent on the TWEAK/Fn14 pathway. J Autoimmun 2013;43:44-54.

18 Chen T, Guo ZP, Li L, Li MM, Wang TT, Jia RZ, Cao N, Li JY: TWEAK enhances E-selectin and ICAM-1 expression, and may contribute to the development of cutaneous vasculitis. PLoS One 2013;8:e56830.

19 Winkles JA, Tran NL, Brown SA, Stains N, Cunliffe HE, Berens ME: Role of TWEAK and Fn14 in tumor biology. Front Biosci 2007;12:2761-2771.

-20 Zhu LX, Zhang HH, Mei YF, Zhao YP, Zhang ZY: Role of tumor necrosis factor-like weak inducer of apoptosis (TWEAK)/fibroblast growth factor-inducible 14 (Fnl4) axis in rheumatic diseases. Chin Med J (Engl) 2012; 125:3898-3904

21 Gao HX, Campbell SR, Burkly LC, Jakubowski A, Jarchum I, Banas B, Saleem MA, Mathieson PW, Berman JW, Michaelson JS, Putterman C: TNF-like weak inducer of apoptosis (TWEAK) induces inflammatory and proliferative effects in human kidney cells. Cytokine 2009;46:24-35.

22 Xia Y, Campbell SR, Broder A, Herlitz L, Abadi M, Wu P, Michaelson JS, Burkly LC, Putterman C: Inhibition of the TWEAK/Fn14 pathway attenuates renal disease in nephrotoxic serum nephritis. Clin Immunol 2012;145:108-121.

23 Xia Y, Herlitz LC, Gindea S, Wen J, Pawar RD, Misharin A, Perlman H, Wu L, Wu P, Michaelson JS, Burkly LC, Putterman C: Deficiency of fibroblast growth factor-inducible 14 (Fn14) preserves the filtration barrier and ameliorates lupus nephritis. J Am Soc Nephrol 2015;26:1053-1070.

-24 Schneider P, Schwenzer R, Haas E, Mühlenbeck F, Schubert G, Scheurich P, Tschopp J, Wajant H: TWEAK can induce cell death via endogenous TNF and TNF receptor 1. Eur J Immunol 1999;29:1785-1792.

-25 Nakayama M, Ishidoh K, Kojima Y, Harada N, Kominami E, Okumura K, Yagita H: Fibroblast growth factorinducible 14 mediates multiple pathways of TWEAK-induced cell death. The Journal of Immunology 2003;170:341-348.

26 Vince JE, Chau D, Callus B, Wong WW, Hawkins CJ, Schneider P, McKinlay M, Benetatos CA, Condon SM, Chunduru SK, Yeoh G, Brink R, Vaux DL, Silke J: TWEAK-FN14 signaling induces lysosomal degradation of a cIAP1-TRAF2 complex to sensitize tumor cells to TNFalpha. J Cell Biol 2008;182:171-184.

27 Ikner A, Ashkenazi A: TWEAK induces apoptosis through a death-signaling complex comprising receptor-interacting protein 1 (RIP1), Fas-associated death domain (FADD), and caspase-8. J Biol Chem 2011;286:21546-21554.

28 Campbell S, Burkly LC, Gao HX, Berman JW, Su L, Browning B, Zheng T, Schiffer L, Michaelson JS, Putterman C: Proinflammatory Effects of Tweak/Fn14 Interactions in Glomerular Mesangial Cells. The Journal of Immunology 2006;176:1889-1898.

29 Vucic D: TRAF2 and cellular IAPs: a critical link in TNFR family signaling. Adv Exp Med Biol 2011;691:6378.

-30 Yang J, Li Y, Liu YQ Long JW, Tian F, Dong J, Shen GX, Tu YT, Tao J: Expression of antiapoptotic protein c-FLIP is upregulated in psoriasis epidermis. Eur J Dermatol 2009;19:29-33.

-31 Doerner J, Chalmers SA, Friedman A, Putterman C: Fn14 deficiency protects lupus-prone mice from histological lupus erythematosus-like skin inflammation induced by ultraviolet light. Exp Dermatol 2016;25:969-976.

32 Di Bari F: Atopic dermatitis and alpha-chemokines. Clin Ter 2015;166:e182-187.

-33 Rebane A, Zimmermann M, Aab A, Baurecht H, Koreck A, Karelson M, Abram K, Metsalu T, Pihlap M, Meyer N, Folster-Holst R, Nagy N, Kemeny L, Kingo K, Vilo J, Illig T, Akdis M, Franke A, Novak N, Weidinger S, Akdis CA: Mechanisms of IFN-gamma-induced apoptosis of human skin keratinocytes in patients with atopic dermatitis. J Allergy Clin Immunol 2012;129:1297-1306.

34 Achtman JC, Werth VP: Pathophysiology of cutaneous lupus erythematosus. Arthritis Res Ther 2015;17:182.

-35 Yoneda K, Demitsu T, Kakurai M, Narita T, Nakai K, Kubota Y, Ishii N, Hashimoto T: Detection of apoptotic keratinocytes in a case of bullous pemphigoid developed after graft-versus-host disease. Acta Derm Venereol 2014;94:231-232.

-36 Coimbra S, Figueiredo A, Castro E, Rocha-Pereira P, Santos-Silva A: The roles of cells and cytokines in the pathogenesis of psoriasis. Int J Dermatol 2012; 51:389-395.

-37 Chelimo C, Wouldes TA, Cameron LD, Elwood JM: Risk factors for and prevention of human papillomaviruses (HPV), genital warts and cervical cancer. J Infect 2013;66:207-217. 


\section{Cellular Physiology Cell Physiol Biochem 2017;43:579-588 and Biochemistry \begin{tabular}{l|l} 
DOI: 10.1159/000480530 & $\begin{array}{l}\text { O 2017 The Author(s). Published by S. Karger AG, Basel } \\
\text { www.karger.com/cpb }\end{array}$
\end{tabular} \\ Wang et al.: Fn14-TRAF2-TNFR Axis}

-38 Chen JJ: Genomic instability induced by human papillomavirus oncogenes. N Am J Med Sci (Boston) 2010;3:43-47.

-39 Tran LS, Bergot AS, Mattarollo SR, Mittal D, Frazer IH: Human papillomavirus e7 oncoprotein transgenic skin develops an enhanced inflammatory response to 2,4-dinitrochlorobenzene by an arginase-1dependent mechanism. J Invest Dermatol 2014;134:2438-2446.

40 Cabal-Hierro L, Lazo PS: Signal transduction by tumor necrosis factor receptors. Cell Signal 2012;24:12971305.

-41 Sedger LM, McDermott MF: TNF and TNF-receptors: From mediators of cell death and inflammation to therapeutic giants - past, present and future. Cytokine Growth Factor Rev 2014;25:453-472.

$\checkmark 42$ Ham B, Fernandez MC, D'Costa Z, Brodt P: The diverse roles of the TNF axi in cancer progression and metastasis. Trends Cancer Res 2016;11:1-27

43 Candel S, de Oliveira S, Lopez-Munoz A, Garcia-Moreno D, Espin-Palazon R, Tyrkalska SD, Cayuela ML, Renshaw SA, Corbalan-Velez R, Vidal-Abarca I, Tsai HJ, Meseguer J, Sepulcre MP, Mulero V: Tnfa signaling through tnfr2 protects skin against oxidative stress-induced inflammation. PLoS Biol 2014;12:e1001855.

44 Chen X, Oppenheim JJ: Targeting TNFR2, an immune checkpoint stimulator and oncoprotein, is a promising treatment for cancer. Sci Signal 2017;10.

45 Cabal-Hierro L, Artime N, Iglesias J, Prado MA, Ugarte-Gil L, Casado P, Fernández-García B, Darnay BG, Lazo PS: A TRAF2 binding independent region of TNFR2 is responsible for TRAF2 depletion and enhancement of cytotoxicity driven by TNFR1. Oncotarget 2014;5:224-236.

-46 Borghi A, Verstrepen L, Beyaert R: TRAF2 multitasking in TNF receptor-induced signaling to NF-kappaB, MAP kinases and cell death. Biochem Pharmacol 2016;116:1-10.

47 Faustman D, Davis M: TNF receptor 2 pathway: drug target for autoimmune diseases. Nat Rev Drug Discov 2010;9:482-493.

48 Burkly LC: Regulation of Tissue Responses: The TWEAK/Fn14 Pathway and Other TNF/TNFR Superfamily Members That Activate Non-Canonical NFkappaB Signaling. Front Immunol 2015;6:92.

49 Brown SA, Richards CM, Hanscom HN, Feng SL, Winkles JA: The Fn14 cytoplasmic tail binds tumournecrosis-factor-receptor-associated factors 1, 2, 3 and 5 and mediates nuclear factor-kappaB activation. Biochem J 2003;371:395-403.

50 Winkles JA: The TWEAK-Fn14 cytokine-receptor axis: discovery, biology and therapeutic targeting. Nat Rev Drug Discov 2008;7:411-425.

51 Mitsuuchi Y, Benetatos CA, Deng Y, Haimowitz T, Beck SC, Arnone MR, Kapoor GS, Seipel ME, Chunduru SK, McKinlay MA, Begley CG, Condon SM: Bivalent IAP antagonists, but not monovalent IAP antagonists, inhibit TNF-mediated NF-kappaB signaling by degrading TRAF2-associated cIAP1 in cancer cells. Cell Death Discov 2017;3:16046.

52 Liu ZC, Zhou QL: Tumor necrosis factor-like weak inducer of apoptosis and its potential roles in lupus nephritis. Inflamm Res 2012;61:277-284.

53 Burkly LC, Michaelson JS, Zheng TS: TWEAK/Fn14 pathway: an immunological switch for shaping tissue responses. Immunol Rev 2011;244: 99-114.

54 Vince JE, Pantaki D, Feltham R, Mace PD, Cordier SM, Schmukle AC, Davidson AJ, Callus BA, Wong WW, Gentle IE, Carter H, Lee EF, Walczak H, Day CL, Vaux DL, Silke J: TRAF2 must bind to cellular inhibitors of apoptosis for tumor necrosis factor (tnf) to efficiently activate nf-\{kappa\}b and to prevent tnf-induced apoptosis. J Biol Chem 2009;284:35906-35915.

55 Burkly LC: TWEAK/Fn14 axis: the current paradigm of tissue injury-inducible function in the midst of complexities. Semin Immunol 2014;26:229-236.

56 Razmara M, Hilliard B, Ziarani AK, Murali R, Yellayi S, Ghazanfar M, Chen YH, Tykocinski ML: Fn14-TRAIL, a chimeric intercellular signal exchanger, attenuates experimental autoimmune encephalomyelitis. Am J Pathol 2009;174:460-474.

57 Aronin A, Amsili S, Prigozhina TB, Tzdaka K, Rachmilewitz J, Shani N, Tykocinski ML, Dranitzki Elhalel M: Fn14*TRAIL effectively inhibits hepatocellular carcinoma growth. PLoS One 2013;8:e77050.

58 Zhou H, Ekmekcioglu S, Marks JW, Mohamedali KA, Asrani K, Phillips KK, Brown SA, Cheng E, Weiss MB, Hittelman WN, Tran NL, Yagita H, Winkles JA, Rosenblum MG: The TWEAK receptor Fn14 is a therapeutic target in melanoma: immunotoxins targeting Fn14 receptor for malignant melanoma treatment. J Invest Dermatol 2013;133:1052-1062. 